\title{
AUTOMORPHISMS OF THICKENINGS
}

\author{
BY J. P. E. HODGSON
}

Communicated by William Browder, April 13, 1967

An $m$-thickening of a CW-complex $K^{k}$ (see [7]) consists of a simple homotopy equivalence $\psi: K_{1^{*}}^{k} \rightarrow M_{1^{*}}$, where $m \geqq k+3$, together with an orientation of the tangent space at the base point. In this note we associate to any PL (or smooth) manifold, semisimplicial complexes corresponding to the homotopy equivalences of the manifold with itself, and to PL (or smooth) automorphisms. By giving a suitable definition of a relative complex we obtain a long exact sequence of homotopy groups. In the case where we restrict our manifolds to be thickenings of certain 'good' complexes we are able to give an interpretation of the relative terms, by means of the structure of certain thickenings. As a consequence we are able to describe the group of quasi-isotopy classes of PL-automorphisms of $S^{p} \times S^{q}$ which are homotopic to the identity. The full details will appear elsewhere. This note may be regarded as bearing much the same relation to [7] as [6] does to [5].

As in [7] the bulk of the material is applicable to both the piecewise linear and smooth categories. The terms homeomorphism, manifold, etc., should therefore be interpreted accordingly.

Definitions and notation. Let $M^{m}$ be a compact connected manifold, and $D_{*}^{m} \subset M^{m}$ a disc containing the base point of $M$, in case $M$ has boundary we suppose $\partial M \cap D_{*}=D_{*}^{m-1} \ni *$. We now define the semisimplicial complexes with which we shall be working.

The complex $\varepsilon(M)$. A $k$-simplex of $\mathcal{E}(M)$ is a homotopy equivalence

$$
F: \Delta^{k} \times M \rightarrow \Delta^{k} \times M
$$

such that

(1) If $\Delta^{s}$ is a face of $\Delta^{k}$ the inclusion induces $F^{\prime}: \Delta^{s} \times M \rightarrow \Delta^{8} \times M$.

(2) $F \mid \Delta^{k} \times D_{*}^{m}=$ Identity.

The complex $\mathscr{D}(M)$. Here it is necessary to make a distinction between the piecewise linear and smooth cases.

Smooth case. A $k$-simplex of $D(M)$ is a map

$$
F: \Delta^{k} \times M \rightarrow \Delta^{k} \times M
$$

such that 
(1) $F \mid \Delta^{k} \times D_{*}^{m}=$ Id.

(2) There is a subdivision (simplicial) of $\Delta^{k}$ with the property that for $\Delta^{s}$ a simplex of the subdivision then $F \mid \Delta^{s} \times M$ is a diffeomorphism onto its image.

(3) If $\Delta^{r}$ is a face of $\Delta^{k}$ then $F \mid \Delta^{r} \times M$ is an $r$-simplex and $F \mid \Delta^{r} \times M$ $\rightarrow \Delta^{r} \times M$.

Note that the definition as formulated is inductive.

Piecewise linear case. A $k$-simplex of $D(M)$ is a piecewise linear automorphism

$$
F: \Delta^{k} \times M \rightarrow \Delta^{k} \times M
$$

such that

(1) $F \mid \Delta^{k} \times D_{*}^{m}=\mathrm{Id}$.

(2) If $\Delta^{s}$ is a face of $\Delta^{k}$ then $F \mid \Delta^{s} \times M$ is a piecewise linear automorphism of $\Delta^{s} \times M$.

In the piecewise linear case $D(M)$ has also been defined by $\mathrm{C}$. Morlet [3].

Proposition 1. The semisimplicial complexes $\mathcal{E}(M)$ and $D(M)$ have homotopy groups and it is possible to define $\pi_{i}(\mathcal{E}(M), D(M))$ in such a way that the following sequence is exact

$$
\rightarrow \pi_{i}(D(M)) \rightarrow \pi_{i}(\mathcal{E}(M)) \rightarrow \pi_{i}(\mathcal{E}(M), \mathfrak{D}(M)) \rightarrow \pi_{i-1}(\mathscr{D}(M)) \rightarrow
$$

The proof is entirely straightforward.

In the smooth case we require the following technical lemma to make the transition between geometry and algebra complete.

LEMMA. It is possible to represent the elements of $\pi_{i}(D(M))$ by smooth diffeomorphisms

$$
F: S^{i} \times M \rightarrow S^{i} \times M .
$$

REMARK. These diffeomorphisms are of course not level-preserving.

The main result. Our main result consists of an interpretation, in geometrical terms, for certain cases of the relative term $\pi_{i}(\mathcal{E}(M), \mathfrak{D}(M))$.

ThEOREM 2. Let $\Psi: K^{k} \rightarrow M^{m}$ be an m-thickening (q.v. [7]) of the finite connected CW-complex $K$. Suppose that $K$ is

(1) $(2 k-m+2)$-connected.

(2) a suspension, thus endowing $J^{m}(K)$ with a multiplication. Then there exists a bijection

$$
\pi_{i}(\mathcal{E}(M), \mathfrak{D}(M)) \rightarrow \mathfrak{J}^{m+i}\left(S^{i} K\right) .
$$


If $N^{m}$ is the trivial-thickening of $K$, and letting $D=$ pseudoisotopy classes of homeomorphisms of $N$ homotopic to the identity, then the bijection gives $D \leftrightarrow J^{m+1}(S K)$.

The principal tool required for the proof of the theorem is the following result. (Which is an extension of a theorem due to Wall.)

Theorem 3. Let $(K, L)$ be a $(2 k-m+1)$-connected $\mathrm{CW}$-pair, and $(M, \partial M)$ a manifold and its nonempty boundary. Suppose given $\lambda: L \rightarrow P^{m-1}$ a thickening of $L$ and $j: P^{m-1} \rightarrow \partial M$ an embedding, then given a $(2 k-m+1)$-connected map

$$
f:(K, L) \rightarrow(P, \partial P) \quad \text { such that } f \mid L=j \circ \lambda
$$

there exists a submanifold $N$ of $M$ and a simple homotopy equivalence $g: K \rightarrow N$, such that the following diagram commutes up to homotopy rel $L$

$$
\begin{aligned}
& K^{\stackrel{g}{\rightarrow}} N \stackrel{k}{\rightarrow} M \\
& \qquad \stackrel{\lambda}{\rightarrow} P \stackrel{j}{\rightarrow} \partial M
\end{aligned}
$$

and $\partial N \cap \partial M=P, k \circ g \sim f(\operatorname{rel} L)$.

Further if $(K, L)$ is $(2 k-m+2)$-connected and $j^{\prime}: P \rightarrow \partial M$ is another embedding of $P$ in $\partial M$ with $J: P \times I \rightarrow \partial M \times I$ a concordance joining $j$ and $j^{\prime}$, then the submanifold pairs $(N, P)(i=1,2)$ corresponding to homotopic $(2 k-m+2)$-connected maps $f_{i}:(K, L)$ $\rightarrow(M, \partial M)$ where $f_{1} \mid L=j \circ \lambda$ and $f_{2} \mid L=j^{\prime} \circ \lambda$ are concordant in $(M, \partial M)$ and the concordance may be chosen to extend $J$.

Application. Piecewise linear case only.

Definition. The homeotopy group of a PL manifold is the group of isotopy classes of homeomorphisms homotopic to the identity. The $q$-homeotopy group is the group of concordance classes of such homeomorphisms. Denote this last by $\mathfrak{F C}(M)$.

Theorem 4. If $2 p \geqq q+4, q \geqq p, p+q \geqq 6$, then

$$
\mathfrak{F}\left(S^{p} \times S^{q}\right)=\operatorname{Image}\left\{\pi_{p}\left(\mathrm{PL}_{q}\right)+\pi_{q}\left(\mathrm{PL}_{p}\right) \stackrel{s}{\rightarrow} \pi_{p}\left(\mathrm{PL}_{q+1}\right)+\pi_{q}\left(\mathrm{PL}_{p+1}\right)\right\} .
$$

The groups $\mathrm{PL}_{q}$ are defined as in [2], [4].

This result (see also [1]) is obtained by considering the homeos of $S^{p}+S^{q}-D^{p+q}$ which is a thickening of $S^{p} \bigvee S^{q}$. 


\section{REFERENCES}

1. W. Browder, Diffeomorphisms of 1-connected manifolds, Trans. Amer. Math. Soc. (to appear).

2. A. Haefliger, Le lissage des immersions. I and II, Mimeographed, Geneva, 1966.

3. C. Morlet, Les voisinages tubulaires des variêtés semilineares, C.R. Acad. Sci. Paris 262 (1966), 740-743.

4. C. P. Rourke and B. J. Sanderson, Block bundles. I, Bull. Amer. Math. Soc. 72 (1966), 1036-1039.

5. C. T. C. Wall, Classification problems in differential topology. I, Topology 2 (1963), 253-261.

6. ㄴ. Classification problems in differential topology. II, Topology 2 (1963), 263272.

7. - Classification problems in differential topology. IV, Topology 5 (1966), 73-94.

University of Pennsylvania 